\title{
Note on the Solution of Riccati's Differential Equation
}

\author{
H. Herbert Howe
}

\author{
(February 4, 1960)
}

\begin{abstract}
Three recurrence formulas are developed, giving the solution of a particular case of Riccati's equation in power series valid in the neighborhoods of $0, \infty$, and an arbitrary point, respectively. The first two were programed for computation on the SEAC.
\end{abstract}

\section{Introduction}

A particular case of Riccati's differential equation is important in the theory of radio propagation. In the notation often used in that subject, omitting subscripts irrelevant in the present paper, the equation is

$$
\frac{d \delta}{d \tau}-2 \delta^{2} \tau+1=0
$$

where $\delta$ will be considered the independent variable, and both variables are complex. The equation may be written

$$
\frac{d \tau}{d \delta}=\frac{1}{2 \delta^{2} \tau-1}
$$

Since this is a first-order differential equation, there should be one constant of integration. If the value of $\tau$ is arbitrarily chosen for some value of $\delta$, the differential equation ought to define $\tau$ for all other values of $\delta$. We may, for example, arbitrarily select $\tau(0)$ or $\tau(\infty)$, and from it derive $\tau$ for all other points.

If there exists in the complex $\delta$-plane any point $P$ such that as we approach it the value of $\delta^{2} \tau$ approaches $\frac{1}{2}$, then the derivative $d \tau / d \delta$ approaches infinity, and $P$ is a singular point of $\tau$. Since $\delta^{2} \tau$ involves both the independent and the dependent variables, the position of $P$ depends upon the particular function $\tau$ which we are studying: e.g., upon the initially selected value $\tau(0)$. It is not immediately obvious that any such $P$ exists, nor that there may not be more than one.

A single-valued function of a complex variable $\delta$, with isolated singular points, can be represented [1] by power series as follows: In the $\delta$-plane, draw concentric circles, one through each singular point, and all centered at the origin. Within the smallest circle, the function is represented by an ascending series in $\delta$ (i.e., there is a constant term, and a series of all positive integral powers of $\delta$ ). This series is absolutely convergent and represents the given function for all values of $\delta$ within the circle. Outside the smallest circle, it diverges. On the circle itself, it may or may not converge. This circle is called the "circle of convergence" for that series; as a special case, its radius may be zero.
Outside the largest circle, the function is represented by a descending series, namely a series consisting of a constant and negative integral powers of $\delta$. It is absolutely convergent outside the circle, divergent inside it, and may or may not converge on it.

In the region between any pair of circles, the function is represented by a series containing all integral powers of $\delta$, both positive and negative, and a different series is required for each intercircle region. Such a series is absolutely convergent in the region between the two circles to which it appertains, may or may not converge on those circles, and diverges outside the specified region.

All of these series together represent the given function at all points of the complex plane, except perhaps those on the various circles. The function exists for all points except the singular points themselves; for other points on the various circles, one of the series may or may not represent the function.

For the function in which we are interested, we at first use the hypothesis that there is one singular point, and that therefore there are two series: The ascending and descending ones, which have a common circle of convergence. A few terms of each series are given by Bremmer [2], and a greater number of terms are given by Johler, Walters, and Lilley [3].

Although one of the two series converges for any value of $\delta$ that does not lie on the common circle of convergence, the rate of convergence is slower the nearer the point is to that circle. For points very near the circle, the converging series will not, in general, provide a practical method of computation. Even within the limits of practical computation, it will be impossible to select any fixed number of terms at which to stop the computation, if given accuracy of the computed result is desired. Rather, the number of terms must be increased as $\delta$ gets nearer the circle.

One could, of course, always compute a fixed number of terms; but there would then be no assurance as to how accurate the result would be, nor as to which series should be used, nor even that either one converged. There is thus need for a formulation of the series which does not rely on expressions with a fixed number of terms. 


\section{Ascending Series}

In 1955 , the present author programed radio applications of (1) on the National Bureau of Standards Electronic Automatic Computer (SEAC). Some of the results have been published [4], but not the formulas. Instead of using a fixed number of terms of the series, recurrence formulas were developed for expressing each term as a quadratic function of the preceding ones. To derive the formulas for the ascending series, we express $\tau$ as a series in $\delta$ :

$$
\tau=\sum_{n=0}^{\infty} b_{n} \delta^{n}
$$

Rewrite Riccati's equation as follows:

$$
\frac{1}{d \tau / d \delta}-2 \delta^{2} \tau+1=0
$$

Insert into (4) the value of $\tau$ given in (3), and in one term change the summation index from $n$ to $m$ :

$$
\frac{1}{\sum_{n=0}^{\infty} n b_{n} \delta^{n-1}}-2 \sum_{m=0}^{\infty} b_{m} \delta^{m+2}+1=0 .
$$

Now clear of fractions, remembering that two absolutely convergent series may be multiplied term by term:

$$
1-2 \sum_{m=0}^{\infty} \sum_{n=0}^{\infty} n b_{n} b_{m} \delta^{m+n+1}+\sum_{n=0}^{\infty} n b_{n} \delta^{n-1}=0 .
$$

In the second term, introduce a new summation index $k$, and eliminate the index $m, k$ being defined by $m=k-n$. To find the summation limits for $n$ and $k$ make a plot of $m$ and $n$; in (6) we are to evaluate the term for each point of the following grid:

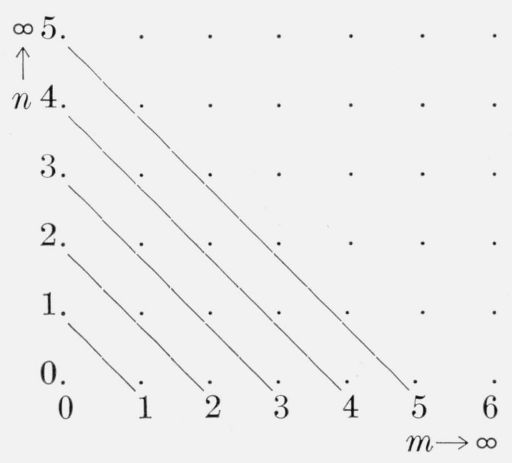

Since $k=m+n$, lines of equal $k$ are the diagonal lines. Since we need to sum for all points of the grid, extending upward and to the right indefinitely, it is seen that we get the same points if we let $k$ run from 0 to $\infty$, and for each value of $k$ let $n$ run from 0 to $k$.

$$
1-2 \sum_{k=0}^{\infty} \sum_{n=0}^{k} n b_{n} b_{k-n} \delta^{k+1}+\sum_{n=0}^{\infty} n b_{n} \delta^{n-1}=0 .
$$

Now change the summation indices again. In the second term, replace $k$ by $j-2$, and in the last term replace $n$ by $j$. Then write out explicitly the terms for $j=0$ and 1 , and collect terms having a common summation:

$$
1+b_{1}+\sum_{j=2}^{\infty}\left[j b_{j}-2 \sum_{n=0}^{j-2} n b_{n} b_{j-n-2}\right] \delta^{j-1}=0 .
$$

Since this equation is to be satisfied for every value of $\delta$ within the circle of convergence, the coefficient of each power of $\delta$ must vanish. This imposes no condition on $b_{o}$, which is equal to $\tau(0)$, which we have previously proposed as the constant of integration. From the constant term of (8),

$$
b_{1}=-1
$$

and for the higher powers of $\delta$, we see that the bracket of (8) must be zero for every value of $j \geqq 2$, i.e.,

$$
b_{j}=(2 / j) \sum_{n=0}^{j-2} n b_{n} b_{j-n-2} .
$$

In particular, $b_{2}=0$.

Now break the right side of (10) into two equal sums, each having a coefficient of $1 / j$; and in one of them replace $n$ by a new summation index $m$ defined by $n=j-2-m$ :

$b_{j}=\frac{1}{j} \sum_{n=0}^{j-2} n b_{n} b_{j-n-2}+\frac{1}{j} \sum_{m=j-2}^{0}(j-2-m) b_{j-2-m} b_{m}$.

Now replace summation-index $m$ by $n$. Since the summations run over the same values of $n$, and since terms with the same $n$ have also the same $b$-product, the series can be combined to give:

$$
b_{j}=\frac{j-2}{j} \sum_{n=0}^{j-2} b_{n} b_{j-n-2} \text { for } j \geqq 2 .
$$

Equations (9) and (12) show the coefficients to be used in (3), after the summation-index of the latter has been changed to $j$.

\section{Recurrence Formulas for the Terms}

For the SEAC computation, it is better to use recurrence formulas involving the terms rather than the coefficients. Let $B_{j}$ represent a term of our series: i.e.,

$$
B_{j}=b_{j} \delta^{j} .
$$

Substituting into (3), (9), and (12), we get

$$
\left.\begin{array}{rl}
\tau & =\sum_{j=0}^{\infty} B_{j} ; B_{1}=-\delta ; B_{2}=0 ; \\
B_{j} & =\frac{j-2}{j} \delta^{2} \sum_{n=0}^{j-2} B_{n} B_{j-n-2} \quad \text { for } \quad j \geqq 3,
\end{array}\right\}
$$

where $B_{0}=b_{0}=\tau(0)$ is the constant of integration, and may be chosen arbitrarily. In practice, it is a boundary condition, chosen to fit the conditions of the problem at hand. 


\section{Descending Series}

Using a similar but somewhat more complicated method, we get for the descending series

$$
\left.\begin{array}{rl}
\tau= & \sum_{j=0}^{\infty} A_{j} ; A_{1}=\left(-\frac{1}{2 A_{0}}\right)\left(\frac{1}{\delta}\right) ; \\
A_{j}= & \left(-\frac{1}{2 A_{0}}\right)\left[\sum_{n=1}^{j-1} A_{n} A_{j-n}\right. \\
& \left.-\frac{j-2}{j} \frac{1}{\delta^{2}} A_{j-2}\right] \quad \text { for } j \geqq 2,
\end{array}\right\}
$$

where $A_{0}=\tau(\infty)$ is the constant of integration.

In order to determine which value of $A_{0}$ belongs to the same function $\tau(\delta)$ as does a given value of $B_{o}$, so that the results of the two series may be combined into a single function, other information about the problem at hand is used [3, pp. 1 to 4 ].

\section{The SEAC Computations}

The SEAC code used (14) and (15) as follows: One of them was selected more or less arbitrarily. The terms $A_{j}$ or $B_{j}$ were computed and saved, since each involves those which come before. When possible, computations were continued until a term was found which was negligibly small. (Real and imaginary parts of $A_{j}$ or $\vec{B}_{j}$ each smaller than, say, $10^{-6}$.) This determination began with $j=3$, since $B_{2}$ is identically zero. When such a term was reached, the computations were discontinued, and the $A$ 's or $B$ 's were summed.

If any $A_{j}$ or $B_{j}$ (starting with $A_{2}$ or $B_{3}$ ) was very large, that computation was abandoned, and the other formula was tried. The same thing was done if no negligibly small term was found within the space available for storing terms in the memory (about 65 terms could be stored). If, by the same criteria, the second formula did not converge, the computation for that particular value of $\tau(0)$ was abandoned. Some of the gaps in the published results [4] come from this cause. (Some occurred because of lack of time to complete the lengthy computations that had been planned.) This might occur either because the point was very near the circle of convergence, so that still more terms were needed; or because for certain functions there are more than one singular point, giving a region in which both series actually diverge.

\section{Convergence of the Series}

The criterion used for terminating the computations (real and imaginary parts of some term both less than $10^{-6}$ ) is not rigorous, and under some circumstances it would be incorrect. This may be most easily studied by substituting into (12) so that each $b_{j}$ is a polynomial in $b_{0}=B_{0}$, to give the equations of [3]. If $B_{0}$ happens to be such that any $b_{j}$ is zero, then $B_{j}$ will be zero regardless of $\delta$, even though subsequent values of $B$ might be of substantial size. For example, two out of every three $b$ 's are zero if $B_{0}=0$; or $b_{7}$ is zero if $5+8 B_{0}^{3}=0$, etc.

For the problem under consideration, each value of $B_{0}$ for which $\tau(\delta)$ was to be derived had an amplitude of $\pi / 3$, and the moduli were all transcendental numbers exceeding $1.8[3$, p. 20]. Being transcendental, they could not make any $b_{j}$ exactly zero; and it is extremely unlikely that, among the small number of values that were used, there would be one that would make any $b$, negligibly small. The expansions of $b_{j}$ through $j=11$ as polynomials in $b_{0}$ [3] show that the coefficients of powers of $b_{0}$ tend to increase as $j$ increases; and since the modulus of $b_{0}$ exceeds unity, it seems most probable that the values of $b_{j}$ continue to increase as $j$ increases. Hence, it seems warranted to conclude that when any $B_{j}$ becomes negligibly small it is probably because $|\delta|$ is less than unity, and $\left|\delta^{j}\right|$ is small; so that succeeding values of $\left|B_{j}\right|$ probably will be still smaller, on account of the factor $\delta^{2}$ in (14).

A similar argument seems to apply to the descending series. More reliable criteria would be to require that each of several consecutive terms of the series be negligibly small.

\section{Bridging the Gap}

Although neither series can be used for practical computation for points near the circle of convergence (or between the circles of convergence, if there is more than one singular point), the function itself is regular in any region not including a singular point; and for a point far from a singular point, the derivatives would not be excessive. Hence, if we are far from a singular point, it may be entirely proper to interpolate across the gap where neither series could be used.

Another method of getting values in this gap is by means of analytic continuation. We can use the ascending series to give a value of $\tau$ for some value of $\delta$ which we may call $q$. It is then possible [1, p. 196] to represent $\tau$ as an ascending series in $\delta-q$, which will be valid within the circle centered at $q$ and passing through the nearest singular point. Such a series, obtained by methods analogous to those already described, is

$$
\left.\begin{array}{l}
\tau=\sum_{j=0}^{\infty} C_{j} ; C_{0}=\tau(q) ; C_{1}=\frac{\delta-q}{2 C_{0} q^{2}-1} ; \\
C_{j}\left(1-2 C_{0} q^{2}\right)=\frac{j-2}{j}(\delta-q)^{2} \sum_{n=0}^{j-2} C_{n} C_{j-n-2} \\
\quad+2 q(\delta-q) \frac{j-1}{j} \sum_{n=0}^{j-1} C_{n} C_{j-n-1}+q^{2} \sum_{n=1}^{j-1} C_{n} C_{j-n}
\end{array}\right\}
$$

No computations have actually been made with this formula. 


\section{References}

[1] See any treatise on theory of a complex variable; e.g., Functions of a complex variable, by Edouard Goursat, translated by Earle Raymond Hedrick and Otto Dunkel, pp. 19, 80-84 (Ginn \& Co., 1916).

[2] H. Bremmer, Terrestrial radio waves. Elsevier, New York, N.Y., 1949 , p. 45 . Bremmer errs in saving that there exists an intermediate region in which $\tau$ may be calculated from either series.

[3] J. R. Johler, L. C. Walters, and C. M. Lilley, Low- and very low-radiofrequency tables of ground wave param- eters for the spherical earth theory: the roots of Riccati's differential equation (supplementary numerical data for NBS circular 573), NBS Tech. Note No. 7, pp. 5-6 (Feb. 1, 1959).

[4] James R. Wait and H. Herbert Howe, Amplitude and phase curves for ground-wave propagation in the band 200 cycles per second to 500 kilocycles, NBS Circ. 574 (1956).

Boulder, Colo. 